\title{
Multiparticle Higgs and vector boson amplitudes at threshold
}

\author{
Valentin V. Khoze \\ Institute for Particle Physics Phenomenology, Department of Physics, Durham University, \\ Durham DH1 3LE, United Kingdom \\ E-mail: valya.khoze@durham.ac.uk
}

ABSTRACT: In a spontaneously broken gauge theory we consider (sub)-processes in which one virtual intermediate state (it can be a Higgs or a gauge field) produces many onshell Higgses and massive vector bosons. In the kinematic regime where all final states are produced on their mass threshold, we show how to compute iteratively all tree-level amplitudes $\mathcal{A}_{1 \rightarrow n+m}$ involving an arbitrary number $n$ of Higgs bosons and $m$ of longitudinal vector bosons in the final state, and list the amplitudes coefficients for up to $n=32$ and $m=32$. We find that these amplitudes exhibit factorial growth not only in the number of scalar fields, but also in the number of longitudinal gauge fields, $\mathcal{A}_{1 \rightarrow n+m} \sim n ! m$ !. This growth is not expected to disappear at loop-level in the fixed-order perturbation theory. We conclude that at energies accessible at the next generation of hadron colliders, such as the $50-100 \mathrm{TeV}$ FCC, where $\sqrt{\hat{s}}$ is sufficient to produce $\gg 1 / \alpha_{W}$ of $W, Z$ and $H$, perturbation theory breaks down when applied to the multiparticle electroweak production, at least near the kinematic multiparticle mass threshold where the electroweak gauge-Higgs sector becomes strongly coupled.

Keywords: Higgs Physics, Spontaneous Symmetry Breaking, Scattering Amplitudes, Standard Model

ARXIV EPRINT: 1404.4876 


\section{Contents}

1 Introduction 1

2 Summing tree graphs at threshold in scalar $\phi^{4}$ theory 2

3 Multiparticle production in the Gauge-Higgs theory 5

4 Conclusions 13

\section{Introduction}

At sufficiently high energies it becomes kinematically possible to produce a high multiplicity final state with $n \sim 1 / \alpha$ particles in the weakly interacting theory. In this case the well-known problem of the factorial divergencies [1] of large orders of perturbation theory now becomes critical as it can affect the high- $n$-point amplitudes already at leading order in the weakly coupled perturbation theory. It was pointed out in $[2,3]$ that the factorial growth could arise from the large numbers of Feynman diagrams contributing to the scattering amplitude $\mathcal{A}_{n}$ at large $n$. This line of reasoning should be robust in any quantum field theory which does not exhibit significant cancellations between the diagrams in computations of on-shell quantities. In particular, in the scalar field theory with $\lambda \phi^{4}$-type interactions tree graphs all have the same sign, and the leading-order high-multiplicity amplitudes acquire the factorial behaviour, $\mathcal{A}_{n} \sim \lambda^{n / 2} n$ ! which (assuming that the amplitudes do not decrease very rapidly in moving off the threshold) leads to the factorial growth of the cross-section, $\sigma_{n}^{\text {tree }} \sim \lambda^{n} n ! \times f_{n}$ (kinematics), in violation of unitarity. This signals the breakdown of perturbative description of these observables for $n>1 / \lambda$.

Multi-particle amplitudes in scalar field theory were studied in depth in the literature in 1990's, see papers [4-14] and references therein. In section 2 we will briefly review their results for the tree-level amplitudes at threshold, relevant for us in this paper. We will also discuss aspects of multi-boson production away from the threshold and the role of the loop corrections in the Conclusions section.

The factorial growth of the amplitudes in scalar QFT is to be contrasted with their behaviour in gauge theory. In the massless gauge theory, for example in $\mathrm{QCD}$, the gauge invariance, on-shell conditions and other symmetries result in dramatic cancellations between Feynman diagrams for on-shell quantities such as scattering amplitudes. As the result there is no manifest factorial growth in amplitudes. For example the maximal helicity violating (MHV) amplitudes (those with 2 negative and $n-2$ positive helicity gluons) are given for any $\mathrm{n}$ by the single-term Parke-Taylor expression [15],

$$
\mathcal{A}_{n}^{\text {tree MHV }}=\frac{\langle r s\rangle^{4}}{\langle 12\rangle\langle 23\rangle \ldots\langle n 1\rangle}
$$


where the two negative-helicity gluons have momenta $p_{r}$ and $p_{s}$; all others are of positive helicity, and $\langle i j\rangle$ is the familiar holomorphic spinor product, see e.g. [16, 17] for a review of the spinor helicity formalism.

The main motivation of this paper is to answer the question of whether the weak sector of the Standard Model (SM), as approximated by the spontaneously broken SU(2) gauge theory, will retain the factorial growth of the multi-particle tree-level amplitudes found in the scalar QFT, or will it follow the regular behaviour of QCD amplitudes. The Gauge-Higgs theory will be analysed in section 3 using the generalisation of the Brown's generating function technique [6] of section 2 .

Another motivation for studying the high-multiplicity production in the electroweak sector at high energies is the close analogy and complementarity between these perturbative processes and the instanton-like processes over the sphaleron barrier [18-25] which, if observable at the next generation of hadron colliders, would violate the Baryon minus Lepton $(B-L)$ number in the SM.

\section{Summing tree graphs at threshold in scalar $\phi^{4}$ theory}

Working at tree level amounts to switching off loop effects controlled by the Planck's constant $\bar{h}$, thus tree-level on-shell amplitudes or currents are essentially classical quantities. As such, they should be governed by classical equations of the system with an appropriate source term added to distinguish between different multi-particle states. An elegant formalism for computing all tree-level multiboson amplitudes at threshold in terms of a classical generating function was introduced by Brown [6] for scalar field theory, which we will briefly review below. Based on solving classical equations directly this approach readily bypasses summation over individual Feynman diagrams.

The amplitude $\mathcal{A}_{1 \rightarrow n}$ for the field $\phi$ to create $n$ particles in the real scalar field theory with the Lagrangian

$$
\mathcal{L}_{\rho}(\phi)=\frac{1}{2}(\partial \phi)^{2}-\frac{1}{2} M^{2} \phi^{2}-\frac{1}{4} \lambda \phi^{4}+\rho \phi,
$$

is derived by differentiating the matrix element of the initial state, $\left\langle 0_{\text {out }}|\phi(0)| 0_{\text {in }}\right\rangle_{\rho}$ with respect to the source $\rho(x)$ and applying the LSZ reduction,

$$
\langle n|\phi(x)| 0\rangle=\lim _{\rho \rightarrow 0}\left[\prod_{j=1}^{n} \lim _{p_{j}^{2} \rightarrow M^{2}} \int d^{4} x_{j} e^{i p_{j} \cdot x_{j}}\left(M^{2}-p_{j}^{2}\right) \frac{\delta}{\delta \rho\left(x_{j}\right)}\right]\left\langle 0_{\text {out }}|\phi(x)| 0_{\text {in }}\right\rangle_{\rho} .
$$

Tree-level approximation is obtained by replacing the matrix element $\left\langle 0_{\text {out }}|\phi(x)| 0_{\text {in }}\right\rangle_{\rho} \longrightarrow$ $\phi_{\mathrm{cl}}(x)$ by a solution to the classical field equation corresponding to the Lagrangian $\mathcal{L}_{\rho}(\phi)$, including the source $\rho(x)$ term. This defines the classical field as the functional of the source, $\phi_{\mathrm{cl}}[\rho]$.

Next step is to go to the threshold limit where all the outgoing particles are produced at rest, $\vec{p}_{j}=0$. In this limit, it is sufficient to consider the spatially-independent source $\rho(t)$. Specifically, before taking the $p_{j}^{2} \rightarrow M^{2}$ limit in (2.2), we set all outgoing momenta 
to $p_{j}^{\mu}=(\omega, \overrightarrow{0})$, and choose $\rho(t)=\rho_{0}(\omega) e^{i \omega t}$. This amounts to the substitution

$$
\left(M^{2}-p_{j}^{2}\right) \frac{\delta}{\delta \rho\left(x_{j}\right)} \longrightarrow\left(M^{2}-\omega^{2}\right) \frac{\delta}{\delta \rho\left(t_{j}\right)}=\frac{\delta}{\delta z\left(t_{j}\right)},
$$

where we defined

$$
z(t):=\frac{\rho_{0}(\omega) e^{i \omega t}}{M^{2}-\omega^{2}-i \epsilon}:=z_{0} e^{i \omega t} .
$$

When the classical solution $\phi_{\mathrm{cl}}$ is re-expressed as the functional of the new variable $z(t)$ rather than the original source $\rho(t)$, one can take the required on-shell limit $\omega \rightarrow M$ simultaneously with sending the amplitude of the source to zero, $\rho_{0}(\omega) \rightarrow 0$ such that $z_{0}$ remains finite [6]. For each external leg operator acting on $\phi_{\mathrm{cl}}$ in (2.2) we have,

$$
\int d^{4} x_{j} e^{i p_{j} \cdot x_{j}}\left(M^{2}-p_{j}^{2}\right) \frac{\delta}{\delta \rho\left(x_{j}\right)} \cdot \phi_{\mathrm{cl}}(t)=e^{i \omega t} \frac{\partial \phi_{\mathrm{cl}}}{\partial z(t)}=\frac{\partial \phi_{\mathrm{cl}}}{\partial z_{0}} .
$$

The tree-level amplitude $\mathcal{A}_{1 \rightarrow n}$ at the $n$-particle threshold is thus given by

$$
\mathcal{A}_{1 \rightarrow n}=\langle n|\phi(0)| 0\rangle=\left.\left(\frac{\partial}{\partial z}\right)^{n} \phi_{\mathrm{cl}}\right|_{z=0},
$$

where the generating function $\phi_{\mathrm{cl}}(z(t))$ is a particular classical solution which we will now determine. As we already noted, $\phi_{\mathrm{cl}}(z(t))$ is unaffected by the double scaling limit, $\omega \rightarrow m$ with $\rho_{0}(\omega) \rightarrow 0$, and at the same time the source term drops out from its defining classical equation. It reduces to an ordinary differential equation for $\phi(t)$ with no source term. For the theory described by the Lagrangian (2.1) it reads,

$$
d_{t}^{2} \phi+M^{2} \phi+\lambda \phi^{3}=0
$$

To give the generating function of amplitudes at multiparticle thresholds, the solution must contain only the positive frequency components of the form $e^{+i n M t}$ where $n$ is the number of final state particles in the amplitude $\mathcal{A}_{1 \rightarrow n}$. This follows immediately from (2.6). Thus, the solution we are after is given by the Taylor expansion in powers of the complex variable $z(t)$,

$$
\phi_{\mathrm{cl}}(t)=z(t)+\sum_{n=2}^{\infty} d_{n} z(t)^{n}
$$

In the limit where interactions are switched off, $\lambda=0$, the correctly normalised solution is $\phi_{\mathrm{cl}}=z(t)$ and this fixes the coefficient of the first term on the r.h.s. of (2.8). As the solution contains only positive frequency harmonics, it is a complex function of Minkowski time. This also fixes the initial conditions of the solution, $\phi_{\mathrm{cl}}(t) \rightarrow 0$ as $\operatorname{Im}(t) \rightarrow \infty$. $\operatorname{In}$ Euclidean time the solution is real.

Coefficients $d_{n}$ determine the actual amplitudes via (2.6),

$$
\mathcal{A}_{1 \rightarrow n}=n ! d_{n},
$$

they can either be read off the classical soluton when it is known, or otherwise be found directly by solving equations of motion iteratively in powers of $z$. 
The classical generating function approach of [6] amounts to finding the $\vec{x}$-independent solution of the Euler-Lagrange equations as an analytic function of $z$ in the form (2.8), and computing the amplitudes via (2.6) or (2.9).

The classical generating function for the theory defined by (2.1) is surprisingly simple and can be written in closed form [6],

$$
\phi_{\mathrm{cl}}(t)=\frac{z(t)}{1-\frac{\lambda}{8 M^{2}} z(t)^{2}} .
$$

It is easily checked that the expression in (2.10) solves the classical equation (2.7) and has the correct form, $\phi_{\mathrm{cl}}=z+\ldots$ as $z \rightarrow 0$. Scattering amplitudes at threshold are then given by $[4,6]$

$$
\mathcal{A}_{1 \rightarrow n}=\left.\left(\frac{\partial}{\partial z}\right)^{n} \phi_{\mathrm{cl}}\right|_{z=0}=n !\left(\frac{\lambda}{8 M^{2}}\right)^{\frac{n-1}{2}} .
$$

We see that the leading order (tree-level) amplitudes in scalar QFT grow factorially with $n$, which ultimately is the consequence of the growth in the number of diagrams in perturbation theory.

The generating functions formalism works equally well also in the real scalar field theory with spontaneously broken $\mathcal{Z}_{2}$ symmetry. For future reference we will denote the scalar field of this model as $h(x)$. The Lagrangian takes the familiar form,

$$
\mathcal{L}(h)=\frac{1}{2}(\partial h)^{2}-\frac{\lambda}{4}\left(h^{2}-v^{2}\right)^{2},
$$

where $v$ is the VEV of $h(x)$. The classical equation for the spatially uniform field $h(t)$,

$$
d_{t}^{2} h=-\lambda h^{3}+\lambda v^{2} h
$$

again has a simple closed-form solution [6]:

$$
h_{\mathrm{cl}}(t)=v \frac{1+\frac{z(t)}{2 v}}{1-\frac{z(t)}{2 v}}
$$

with the correct form of initial conditions, $h_{\mathrm{cl}}=v+z+\ldots$ as $z \rightarrow 0$. The Taylor expansion of the generating function (2.14) can be recast in the form (cf. (2.8)),

$$
h_{\mathrm{cl}}(t)=2 v \sum_{n=0}^{\infty}\left(\frac{z(t)}{2 v}\right)^{n} d_{n}=v+2 \sum_{n=1}^{\infty}\left(\frac{z(t)}{2 v}\right)^{n},
$$

i.e. with $d_{0}=1 / 2$ and all $d_{n \geq 1}=1$. Scattering amplitudes at threshold in this theory are given by $[5,6]$

$$
\mathcal{A}_{1 \rightarrow n}=\left.\left(\frac{\partial}{\partial z}\right)^{n} h_{\mathrm{cl}}\right|_{z=0}=n !(2 v)^{1-n} .
$$

This theory of a single real scalar field with a spontaneously broken $h \rightarrow-h$ discrete symmetry is a toy version of the Higgs sector of the SM. The field $h$ looks lie the SM Higgs in the unitary gauge, but without the accompaniment of longitudinal massive vector bosons. In the following section we will apply the generating functions method to the spontaneously broken gauge theory and examine if the factorial growth, which is manifest in (2.16), also persists in the Gauge-Higgs theory. 


\section{Multiparticle production in the Gauge-Higgs theory}

For concreteness we will concentrate on the simplest Non-Abelian case of interest - the $\mathrm{SU}(2)$ gauge theory spontaneously broken by the vacuum expectation value $v$ of the Higgs doublet,

$$
\mathcal{L}=-\frac{1}{4} F^{a \mu \nu} F_{\mu \nu}^{a}+\left|D_{\mu} H\right|^{2}-\lambda\left(|H|^{2}-\frac{v^{2}}{2}\right)^{2} .
$$

This theory describes the weak sector of the SM in the limit of the vanishing $\theta_{\mathrm{W}}$. In the unitary gauge,

$$
H=\frac{1}{\sqrt{2}}(0, h)
$$

and the Higgs potential in terms of $h$ takes the same form as in eq. (2.12). The particle content of the model is given by the neutral Higgs state, $h$, and a triplet of massive vector bosons, $W^{ \pm}$and $Z^{0}$, described by $A_{\mu}^{a}$ with $a=1,2,3$, which we will collectively refer to as $V$. The Higgs mass and the mass of the vector boson triplet are given by,

$$
M_{h}=\sqrt{2 \lambda} v \simeq 125.66 \mathrm{GeV}, \quad M_{V}=\frac{g v}{2} \simeq 80.384 \mathrm{GeV},
$$

where we have also shown their numerical values, set by the SM Higgs and $W$ boson masses, which will be uses in our calculations of the amplitudes below.

The kinematic regime of interest is when a single virtual state - the Higgs or a gauge boson, decays into $n$ Higgs bosons and $m$ vector bosons, all with vanishing spatial momenta. In the rest frame of the initial virtual boson we have

$$
p_{\text {in }}^{\mu}=\left(n M_{h}+m M_{V}, \overrightarrow{0}\right) \rightarrow \sum_{j=1}^{n} p_{j}^{\mu}+\sum_{k=1}^{m} p_{k}^{\mu}, \quad \text { where } \quad p_{j}^{\mu}=\left(M_{h}, \overrightarrow{0}\right), \quad p_{k}^{\mu}=\left(M_{V}, \overrightarrow{0}\right) .
$$

This system can be Lorentz boosted, giving all momenta a non-vanishing $p^{3}$ component. The process in this frame corresponds to the highly virtual boson being produced in the $p p$ collision (e.g. the gluon gluon fusion to a Higgs) which decays to a maximal kinematically allowed number of Higgses and massive vector bosons. The boosted direction along $p^{3}$ is the longitudinal direction. All momenta in the boosted frame have the form, $p^{\mu}=\left(p^{0}, 0,0, p^{3}\right)$.

For the rest of the analysis we return to the rest frame (3.4) where $p^{3}$ is vanishing (or infinitesimally small). The transversality condition, $p^{\mu} A_{\mu}^{a}=0$, allows us to set $A_{0}^{a}=0$. We thus are left with the following degrees of freedom: $\left\{h(t), A_{m}^{a}(t)\right\}$ with $m=1,2,3$, the first two being the transverse and the third - the longitudinal polarisations of the triplet $(a=1,2,3)$ of massive vector bosons.

The Lagrangian (3.1) reduced on these spatially-independent components reads,

$$
\mathcal{L}=\frac{1}{2}\left(d_{t} A_{m}^{a}\right)^{2}+\frac{1}{2}\left(d_{t} h\right)^{2}-\frac{g^{2}}{8} h^{2}\left(A_{m}^{a}\right)^{2}-\frac{g^{2}}{4}\left(\left(A_{m}^{a}\right)^{2}\left(A_{n}^{b}\right)^{2}-\left(A_{m}^{a} A_{n}^{a}\right)^{2}\right)-\frac{\lambda}{4}\left(h^{2}-v^{2}\right)^{2}
$$

and the equations of motion for $h_{\mathrm{cl}}(t)$ and $A_{m \mathrm{cl}}^{a}(t)$ are,

$$
\begin{aligned}
d_{t}^{2} h & =-\lambda h^{3}+\lambda v^{2} h-\frac{g^{2}}{4}\left(A_{m}^{a}\right)^{2} h, \\
d_{t}^{2} A_{m}^{a} & =-\frac{g^{2}}{4} h^{2} A_{m}^{a}-g^{2}\left(\left(A_{n}^{b}\right)^{2} A_{m}^{a}-\left(A_{n}^{a} A_{n}^{b}\right) A_{m}^{b}\right)
\end{aligned}
$$


This system of equations can now be solved iteratively.

To simplify the derivation we will assume that the final state does not contain transverse polarisations of the vector bosons, and concentrate on the production of longitudinal polarisations, $A_{3}^{a}$ and Higgses $h$. The 'commutator' term in (3.5) and (3.7) then drops out and we get,

$$
\begin{aligned}
d_{t}^{2} h & =-\lambda h^{3}+\lambda v^{2} h-\frac{g^{2}}{4}\left(A_{L}^{a}\right)^{2} h, \\
d_{t}^{2} A_{L}^{a} & =-\frac{g^{2}}{4} h^{2} A_{L}^{a} .
\end{aligned}
$$

The classical solutions required to give the generating function of the amplitudes on the multi- $h$, multi- $V_{L}$ threshold, should be the analytic functions (i.e. given by the double Taylor expansion in terms) of two variables,

$$
z(t)=z_{0} e^{i M_{h} t}, \quad w^{a}(t)=w_{0}^{a} e^{i M_{V} t},
$$

with the leading-order terms being,

$$
h_{\mathrm{cl}}(t)=v+z(t)+\ldots, \quad A_{L \mathrm{cl}}^{a}(t)=w^{a}(t)+\ldots,
$$

The system of equations (3.8)-(3.9) can be shown to depend only on a single parameter, by re-writing them in terms of dimensionless variabeles, $t=M_{h} t, h=h / v$ and $A_{L}^{a}=A_{L}^{a} / v$,

$$
\begin{aligned}
d_{t}^{2} h & =-\frac{1}{2}\left(h^{3}-h\right)-\kappa^{2}\left(A_{L}^{a}\right)^{2} h, \\
d_{t}^{2} A_{L}^{a} & =-\kappa^{2} h^{2} A_{L}^{a},
\end{aligned}
$$

with

$$
\kappa:=\frac{g}{2 \sqrt{2 \lambda}}=\frac{M_{V}}{M_{h}} .
$$

Before we write down the double Taylor expansion of the generating functions $h_{\mathrm{cl}}$ and $A_{L \mathrm{cl}}^{a}$, we define the scalar function $\mathrm{A}_{\mathrm{cl}}$ for vector bosons via

$$
A_{L \mathrm{cl}}^{a}=w^{a} \mathrm{~A}_{\mathrm{cl}}
$$

and introduce the new combination,

$$
W=w^{a} w^{a} .
$$

We now write in the double Taylor expansion in the form,

$$
\begin{aligned}
& h_{\mathrm{cl}}(z, W)=\sum_{n=0}^{\infty} \sum_{k=0}^{\infty} d_{n, k} z^{n} W^{k}, \quad \text { with } d_{0,0}=1 \text { and } d_{1,0}=1, \\
& \mathrm{~A}_{\mathrm{cl}}(z, W)=\sum_{n=0}^{\infty} \sum_{k=0}^{\infty} a_{n, k} z^{n} W^{k}, \quad \text { with } a_{0,0}=1 .
\end{aligned}
$$


Differentiating these expressions twice with $t$ we write down the equations (3.12)-(3.13) in the form, ${ }^{1}$

$$
\begin{aligned}
d_{n, k}(n+2 \kappa k)^{2} z^{n} W^{k} & =\left.\left[\frac{1}{2}\left(h^{3}-h\right)+\kappa^{2} W \mathrm{~A}^{2} h\right]\right|_{z^{n} W^{k}}, \\
a_{n, k}(n+\kappa+2 \kappa k)^{2} z^{n} W^{k} & =\left.\left[\kappa^{2} h^{2} \mathrm{~A}\right]\right|_{z^{n} W^{k}} .
\end{aligned}
$$

These equations are solved iteratively as follows. First we set $k=0$ and solve the Higgs equations (3.19) for all values of $n$ thus determining all coefficients ${ }^{2} d_{n, 0}$. No other coefficients enter this equation for $k=0$. Then we solve the $A$-equations (3.20) for the coefficients $a_{n, 0}$ for each $n$. Next we set $k=1$, and solve equations (3.19) for all $n$ to determine $d_{n, 1}$. Following this, the coefficients $a_{n, 1}$ are found by solving (3.20) at $k=1$ for all values of $n$. This procedure is repeated for all values of $k$.

After implementing this iterative algorithm in Mathematica we can solve for $d_{n, k}$ and $a_{n, k}$ to any desired values of $n$ and $k$ numerically.

There is not much hope to find a simple analytical solution for the generating functions as was the case in the scalar field theory; even at $k=0$ the $a_{n, 0}$ coefficients start becoming increasingly complicated already at relatively low values of $n$,

$$
\begin{aligned}
& a_{4,0}=\frac{\kappa^{2}\left(9+33 \kappa+75 \kappa^{2}+90 \kappa^{3}+64 \kappa^{4}+24 \kappa^{5}+4 \kappa^{6}\right)}{24(1+\kappa)(2+\kappa)(1+2 \kappa)(3+2 \kappa)} \\
& a_{5,0}=\frac{\kappa^{2}\left(90+375 \kappa+987 \kappa^{2}+1500 \kappa^{3}+1474 \kappa^{4}+920 \kappa^{5}+360 \kappa^{6}+80 \kappa^{7}+8 \kappa^{8}\right)}{120(1+\kappa)(2+\kappa)(1+2 \kappa)(3+2 \kappa)(5+2 \kappa)}
\end{aligned}
$$

However, the closed form solution is not really needed in order to determine amplitudes at threshold as they are described by the coefficients $d_{n, k}$ and $a_{n, k}$ which are computed straightforwardly in our iterative procedure, as described above.

Before we list the coefficients we solved for, we re-write the double Taylor expansion of the generating functions back in terms of physical dimensionful variables, with an additional rescaling by factors of 2 - to make the comparison with eq. (2.15) more manifest. We define the rescaled coeffiecients

$$
d(n, 2 k):=2^{n+2 k-1} d_{n, k}, \quad a(n, 2 k):=2^{n+2 k} a_{n, k},
$$

and write down the double Taylor expansion of the generating functions in terms of these as follows,

$$
\begin{aligned}
h_{\mathrm{cl}}\left(z, w^{a}\right) & =2 v \sum_{n=0}^{\infty} \sum_{k=0}^{\infty} d(n, 2 k)\left(\frac{z}{2 v}\right)^{n}\left(\frac{w^{a} w^{a}}{(2 v)^{2}}\right)^{k}, \\
A_{L \mathrm{cl}}^{a}\left(z, w^{a}\right) & =w^{a} \sum_{n=0}^{\infty} \sum_{k=0}^{\infty} a(n, 2 k) z^{n}\left(\frac{z}{2 v}\right)^{n}\left(\frac{w^{a} w^{a}}{(2 v)^{2}}\right)^{k},
\end{aligned}
$$

where $z(t)$ and $w^{a}(t)$ are given by eqs. (3.10).

\footnotetext{
${ }^{1}$ Recall that in terms of our dimensionless variables, $z(t)=z_{0} e^{i t}, w(t)=w_{0} e^{i \kappa t}$ and $W=w_{0}^{2} e^{i 2 \kappa t}$.

${ }^{2}$ Not surprisingly, we find $d_{n \geq 1,0}=1 / 2^{n-1}$ in accordance with $(2.15)$.
} 


\begin{tabular}{|c|c|c|c|c|c|c|c|c|c|}
\hline$d(n, m)$ & $\mathrm{m}=0$ & 2 & 4 & 6 & 8 & 10 & 12 & 14 & 16 \\
\hline $\mathrm{n}=0$ & $\frac{1}{2}$ & 1.285 & 1.462 & 1.604 & 1.763 & 1.937 & 2.127 & 2.336 & 2.561 \\
\hline 1 & 1 & 2.508 & 4.304 & 6.422 & 8.914 & $1.183 \times 10^{1}$ & $1.523 \times 10^{1}$ & $1.917 \times 10^{1}$ & $2.373 \times 10^{1}$ \\
\hline 2 & 1 & 3.69 & 8.53 & $1.607 \times 10^{1}$ & $2.696 \times 10^{1}$ & $4.196 \times 10^{1}$ & $6.199 \times 10^{1}$ & $8.809 \times 10^{1}$ & $1.215 \times 10^{2}$ \\
\hline 3 & 1 & 4.868 & $1.414 \times 10^{1}$ & $3.215 \times 10^{1}$ & $6.327 \times 10^{1}$ & $1.131 \times 10^{2}$ & $1.886 \times 10^{2}$ & $2.988 \times 10^{2}$ & $4.545 \times 10^{2}$ \\
\hline 4 & 1 & 6.045 & $2.112 \times 10^{1}$ & $5.628 \times 10^{1}$ & $1.271 \times 10^{2}$ & $2.566 \times 10^{2}$ & $4.772 \times 10^{2}$ & $8.338 \times 10^{2}$ & $1.387 \times 10^{3}$ \\
\hline 5 & 1 & 7.22 & $2.947 \times 10^{1}$ & $9.006 \times 10^{1}$ & $2.297 \times 10^{2}$ & $5.167 \times 10^{2}$ & $1.06 \times 10^{3}$ & $2.027 \times 10^{3}$ & $3.661 \times 10^{3}$ \\
\hline 6 & 1 & 8.394 & $3.92 \times 10^{1}$ & $1.351 \times 10^{2}$ & $3.839 \times 10^{2}$ & $9.528 \times 10^{2}$ & $2.138 \times 10^{3}$ & $4.439 \times 10^{3}$ & $8.656 \times 10^{3}$ \\
\hline 7 & 1 & 9.568 & $5.031 \times 10^{1}$ & $1.93 \times 10^{2}$ & $6.047 \times 10^{2}$ & $1.641 \times 10^{3}$ & $4 . \times 10^{3}$ & $8.962 \times 10^{3}$ & $1.876 \times 10^{4}$ \\
\hline 8 & 1 & $1.074 \times 10^{1}$ & $6.278 \times 10^{1}$ & $2.654 \times 10^{2}$ & $9.089 \times 10^{2}$ & $2.678 \times 10^{3}$ & $7.045 \times 10^{3}$ & $1.695 \times 10^{4}$ & $3.792 \times 10^{4}$ \\
\hline 9 & 1 & $1.191 \times 10^{1}$ & $7.662 \times 10^{1}$ & $3.538 \times 10^{2}$ & $1.315 \times 10^{3}$ & $4.181 \times 10^{3}$ & $1.181 \times 10^{4}$ & $3.035 \times 10^{4}$ & $7.229 \times 10^{4}$ \\
\hline 10 & 1 & $1.309 \times 10^{1}$ & $9.184 \times 10^{1}$ & $4.599 \times 10^{2}$ & $1.844 \times 10^{3}$ & $6.292 \times 10^{3}$ & $1.898 \times 10^{4}$ & $5.194 \times 10^{4}$ & $1.312 \times 10^{5}$ \\
\hline 11 & 1 & $1.426 \times 10^{1}$ & $1.084 \times 10^{2}$ & $5.853 \times 10^{2}$ & $2.517 \times 10^{3}$ & $9.177 \times 10^{3}$ & $2.947 \times 10^{4}$ & $8.55 \times 10^{4}$ & $2.283 \times 10^{5}$ \\
\hline 12 & 1 & $1.543 \times 10^{1}$ & $1.264 \times 10^{2}$ & $7.316 \times 10^{2}$ & $3.36 \times 10^{3}$ & $1.303 \times 10^{4}$ & $4.437 \times 10^{4}$ & $1.361 \times 10^{5}$ & $3.832 \times 10^{5}$ \\
\hline 13 & 1 & $1.66 \times 10^{1}$ & $1.457 \times 10^{2}$ & $9.003 \times 10^{2}$ & $4.398 \times 10^{3}$ & $1.809 \times 10^{4}$ & $6.508 \times 10^{4}$ & $2.104 \times 10^{5}$ & $6.228 \times 10^{5}$ \\
\hline 14 & 1 & $1.777 \times 10^{1}$ & $1.664 \times 10^{2}$ & $1.093 \times 10^{3}$ & $5.66 \times 10^{3}$ & $2.459 \times 10^{4}$ & $9.327 \times 10^{4}$ & $3.17 \times 10^{5}$ & $9.843 \times 10^{5}$ \\
\hline 15 & 1 & $1.894 \times 10^{1}$ & $1.885 \times 10^{2}$ & $1.312 \times 10^{3}$ & $7.175 \times 10^{3}$ & $3.285 \times 10^{4}$ & $1.31 \times 10^{5}$ & $4.669 \times 10^{5}$ & $1.517 \times 10^{6}$ \\
\hline 16 & 1 & $2.011 \times 10^{1}$ & $2.119 \times 10^{2}$ & $1.557 \times 10^{3}$ & $8.974 \times 10^{3}$ & $4.319 \times 10^{4}$ & $1.805 \times 10^{5}$ & $6.737 \times 10^{5}$ & $2.287 \times 10^{6}$ \\
\hline 17 & 1 & $2.129 \times 10^{1}$ & $2.367 \times 10^{2}$ & $1.832 \times 10^{3}$ & $1.109 \times 10^{4}$ & $5.597 \times 10^{4}$ & $2.449 \times 10^{5}$ & $9.543 \times 10^{5}$ & $3.378 \times 10^{6}$ \\
\hline 18 & 1 & $2.246 \times 10^{1}$ & $2.629 \times 10^{2}$ & $2.137 \times 10^{3}$ & $1.357 \times 10^{4}$ & $7.161 \times 10^{4}$ & $3.272 \times 10^{5}$ & $1.33 \times 10^{6}$ & $4.9 \times 10^{6}$ \\
\hline 19 & 1 & $2.363 \times 10^{1}$ & $2.904 \times 10^{2}$ & $2.475 \times 10^{3}$ & $1.643 \times 10^{4}$ & $9.057 \times 10^{4}$ & $4.314 \times 10^{5}$ & $1.825 \times 10^{6}$ & $6.991 \times 10^{6}$ \\
\hline 20 & 1 & $2.48 \times 10^{1}$ & $3.193 \times 10^{2}$ & $2.846 \times 10^{3}$ & $1.972 \times 10^{4}$ & $1.133 \times 10^{5}$ & $5.62 \times 10^{5}$ & $2.471 \times 10^{6}$ & $9.823 \times 10^{6}$ \\
\hline 21 & 1 & $2.597 \times 10^{1}$ & $3.496 \times 10^{2}$ & $3.252 \times 10^{3}$ & $2.349 \times 10^{4}$ & $1.405 \times 10^{5}$ & $7.238 \times 10^{5}$ & $3.303 \times 10^{6}$ & $1.361 \times 10^{7}$ \\
\hline 22 & 1 & $2.714 \times 10^{1}$ & $3.813 \times 10^{2}$ & $3.695 \times 10^{3}$ & $2.777 \times 10^{4}$ & $1.726 \times 10^{5}$ & $9.227 \times 10^{5}$ & $4.364 \times 10^{6}$ & $1.862 \times 10^{7}$ \\
\hline 23 & 1 & $2.831 \times 10^{1}$ & $4.143 \times 10^{2}$ & $4.177 \times 10^{3}$ & $3.261 \times 10^{4}$ & $2.103 \times 10^{5}$ & $1.165 \times 10^{6}$ & $5.705 \times 10^{6}$ & $2.517 \times 10^{7}$ \\
\hline 24 & 1 & $2.948 \times 10^{1}$ & $4.487 \times 10^{2}$ & $4.699 \times 10^{3}$ & $3.806 \times 10^{4}$ & $2.543 \times 10^{5}$ & $1.459 \times 10^{6}$ & $7.384 \times 10^{6}$ & $3.366 \times 10^{7}$ \\
\hline 25 & 1 & $3.065 \times 10^{1}$ & $4.845 \times 10^{2}$ & $5.262 \times 10^{3}$ & $4.417 \times 10^{4}$ & $3.054 \times 10^{5}$ & $1.811 \times 10^{6}$ & $9.471 \times 10^{6}$ & $4.454 \times 10^{7}$ \\
\hline 26 & 1 & $3.182 \times 10^{1}$ & $5.216 \times 10^{2}$ & $5.869 \times 10^{3}$ & $5.098 \times 10^{4}$ & $3.644 \times 10^{5}$ & $2.232 \times 10^{6}$ & $1.204 \times 10^{7}$ & $5.84 \times 10^{7}$ \\
\hline 27 & 1 & $3.299 \times 10^{1}$ & $5.601 \times 10^{2}$ & $6.52 \times 10^{3}$ & $5.854 \times 10^{4}$ & $4.322 \times 10^{5}$ & $2.731 \times 10^{6}$ & $1.519 \times 10^{7}$ & $7.588 \times 10^{7}$ \\
\hline 28 & 1 & $3.416 \times 10^{1}$ & $5.999 \times 10^{2}$ & $7.219 \times 10^{3}$ & $6.692 \times 10^{4}$ & $5.097 \times 10^{5}$ & $3.32 \times 10^{6}$ & $1.902 \times 10^{7}$ & $9.778 \times 10^{7}$ \\
\hline 29 & 1 & $3.533 \times 10^{1}$ & $6.412 \times 10^{2}$ & $7.965 \times 10^{3}$ & $7.617 \times 10^{4}$ & $5.979 \times 10^{5}$ & $4.012 \times 10^{6}$ & $2.365 \times 10^{7}$ & $1.25 \times 10^{8}$ \\
\hline 30 & 1 & $3.65 \times 10^{1}$ & $6.838 \times 10^{2}$ & $8.761 \times 10^{3}$ & $8.634 \times 10^{4}$ & $6.98 \times 10^{5}$ & $4.819 \times 10^{6}$ & $2.921 \times 10^{7}$ & $1.587 \times 10^{8}$ \\
\hline 31 & 1 & $3.767 \times 10^{1}$ & $7.278 \times 10^{2}$ & $9.608 \times 10^{3}$ & $9.75 \times 10^{4}$ & $8.11 \times 10^{5}$ & $5.757 \times 10^{6}$ & $3.586 \times 10^{7}$ & $2 . \times 10^{8}$ \\
\hline 32 & 1 & $3.884 \times 10^{1}$ & $7.731 \times 10^{2}$ & $1.051 \times 10^{4}$ & $1.097 \times 10^{5}$ & $9.382 \times 10^{5}$ & $6.842 \times 10^{6}$ & $4.376 \times 10^{7}$ & $2.505 \times 10^{8}$ \\
\hline
\end{tabular}

Figure 1. Coefficients $d(n, m)$ for $n=0 \ldots 32$ and $m=0 \ldots 16$ of the virtual Higgs decay amplitudes $\mathcal{A}\left(h \rightarrow n \times h+m \times V_{L}\right)=n ! m ! d(n, m)(2 v)^{1-n-m}$ at the $(n+m)$-particle threshold. $V_{L}$ are the longitudinal components of the $W^{ \pm}, Z^{0}$ massive $\mathrm{SU}(2)$ vector bosons. The value of $\kappa$ is set to the SM value, $\kappa=M_{W} / M_{h}=80.384 / 125.66 \simeq 0.6397$. 


\begin{tabular}{|c|c|c|c|c|c|c|c|c|}
\hline$d(n, m)$ & $\mathrm{m}=18$ & 20 & 22 & 24 & 26 & 28 & 30 & 32 \\
\hline $\mathrm{n}=0$ & 2.807 & 3.061 & 3.17 & 3.832 & $2.598 \times 10^{-1}$ & -4.428 & $-1.138 \times 10^{1}$ & $5.89 \times 10^{1}$ \\
\hline 1 & $2.9 \times 10^{1}$ & $3.509 \times 10^{1}$ & $4.218 \times 10^{1}$ & $4.969 \times 10^{1}$ & $6.01 \times 10^{1}$ & $7.468 \times 10^{1}$ & $6.53 \times 10^{1}$ & $2.553 \times 10^{2}$ \\
\hline 2 & $1.637 \times 10^{2}$ & $2.162 \times 10^{2}$ & $2.812 \times 10^{2}$ & $3.607 \times 10^{2}$ & $4.573 \times 10^{2}$ & $5.735 \times 10^{2}$ & $7.114 \times 10^{2}$ & $8.699 \times 10^{2}$ \\
\hline 3 & $6.694 \times 10^{2}$ & $9.601 \times 10^{2}$ & $1.347 \times 10^{3}$ & $1.854 \times 10^{3}$ & $2.511 \times 10^{3}$ & $3.353 \times 10^{3}$ & $4.419 \times 10^{3}$ & $5.755 \times 10^{3}$ \\
\hline 4 & $2.218 \times 10^{3}$ & $3.433 \times 10^{3}$ & $5.169 \times 10^{3}$ & $7.602 \times 10^{3}$ & $1.096 \times 10^{4}$ & $1.551 \times 10^{4}$ & $2.162 \times 10^{4}$ & $2.972 \times 10^{4}$ \\
\hline 5 & $6.319 \times 10^{3}$ & $1.05 \times 10^{4}$ & $1.689 \times 10^{4}$ & $2.643 \times 10^{4}$ & $4.039 \times 10^{4}$ & $6.044 \times 10^{4}$ & $8.881 \times 10^{4}$ & $1.284 \times 10^{5}$ \\
\hline 6 & $1.604 \times 10^{4}$ & $2.847 \times 10^{4}$ & $4.875 \times 10^{4}$ & $8.09 \times 10^{4}$ & $1.307 \times 10^{5}$ & $2.061 \times 10^{5}$ & $3.184 \times 10^{5}$ & $4.827 \times 10^{5}$ \\
\hline 7 & $3.716 \times 10^{4}$ & $7.021 \times 10^{4}$ & $1.275 \times 10^{5}$ & $2.237 \times 10^{5}$ & $3.808 \times 10^{5}$ & $6.316 \times 10^{5}$ & $1.023 \times 10^{6}$ & $1.624 \times 10^{6}$ \\
\hline 8 & $7.992 \times 10^{4}$ & $1.602 \times 10^{5}$ & $3.074 \times 10^{5}$ & $5.686 \times 10^{5}$ & $1.018 \times 10^{6}$ & $1.771 \times 10^{6}$ & $3.003 \times 10^{6}$ & $4.978 \times 10^{6}$ \\
\hline 9 & $1.616 \times 10^{5}$ & $3.424 \times 10^{5}$ & $6.929 \times 10^{5}$ & $1.347 \times 10^{6}$ & $2.53 \times 10^{6}$ & $4.607 \times 10^{6}$ & $8.163 \times 10^{6}$ & $1.411 \times 10^{7}$ \\
\hline 10 & $3.101 \times 10^{5}$ & $6.926 \times 10^{5}$ & $1.474 \times 10^{6}$ & $3.007 \times 10^{6}$ & $5.91 \times 10^{6}$ & $1.124 \times 10^{7}$ & $2.077 \times 10^{7}$ & $3.738 \times 10^{7}$ \\
\hline 11 & $5.689 \times 10^{5}$ & $1.336 \times 10^{6}$ & $2.983 \times 10^{6}$ & $6.37 \times 10^{6}$ & $1.308 \times 10^{7}$ & $2.595 \times 10^{7}$ & $4.992 \times 10^{7}$ & $9.339 \times 10^{7}$ \\
\hline 12 & $1.004 \times 10^{6}$ & $2.473 \times 10^{6}$ & $5.78 \times 10^{6}$ & $1.289 \times 10^{7}$ & $2.762 \times 10^{7}$ & $5.705 \times 10^{7}$ & $1.141 \times 10^{8}$ & $2.216 \times 10^{8}$ \\
\hline 13 & $1.712 \times 10^{6}$ & $4.415 \times 10^{6}$ & $1.078 \times 10^{7}$ & $2.508 \times 10^{7}$ & $5.593 \times 10^{7}$ & $1.201 \times 10^{8}$ & $2.493 \times 10^{8}$ & $5.02 \times 10^{8}$ \\
\hline 14 & $2.832 \times 10^{6}$ & $7.63 \times 10^{6}$ & $1.943 \times 10^{7}$ & $4.707 \times 10^{7}$ & $1.091 \times 10^{8}$ & $2.433 \times 10^{8}$ & $5.236 \times 10^{8}$ & $1.092 \times 10^{9}$ \\
\hline 15 & $4.56 \times 10^{6}$ & $1.281 \times 10^{7}$ & $3.397 \times 10^{7}$ & $8.556 \times 10^{7}$ & $2.059 \times 10^{8}$ & $4.76 \times 10^{8}$ & $1.061 \times 10^{9}$ & $2.287 \times 10^{9}$ \\
\hline 16 & $7.168 \times 10^{6}$ & $2.097 \times 10^{7}$ & $5.78 \times 10^{7}$ & $1.511 \times 10^{8}$ & $3.771 \times 10^{8}$ & $9.026 \times 10^{8}$ & $2.08 \times 10^{9}$ & $4.635 \times 10^{9}$ \\
\hline 17 & $1.102 \times 10^{7}$ & $3.353 \times 10^{7}$ & $9.592 \times 10^{7}$ & $2.6 \times 10^{8}$ & $6.719 \times 10^{8}$ & $1.663 \times 10^{9}$ & $3.961 \times 10^{9}$ & $9.11 \times 10^{9}$ \\
\hline 18 & $1.662 \times 10^{7}$ & $5.248 \times 10^{7}$ & $1.557 \times 10^{8}$ & $4.369 \times 10^{8}$ & $1.168 \times 10^{9}$ & $2.987 \times 10^{9}$ & $7.342 \times 10^{9}$ & $1.741 \times 10^{10}$ \\
\hline 19 & $2.461 \times 10^{7}$ & $8.056 \times 10^{7}$ & $2.474 \times 10^{8}$ & $7.184 \times 10^{8}$ & $1.984 \times 10^{9}$ & $5.237 \times 10^{9}$ & $1.327 \times 10^{10}$ & $3.243 \times 10^{10}$ \\
\hline 20 & $3.585 \times 10^{7}$ & $1.215 \times 10^{8}$ & $3.86 \times 10^{8}$ & $1.158 \times 10^{9}$ & $3.3 \times 10^{9}$ & $8.983 \times 10^{9}$ & $2.346 \times 10^{10}$ & $5.9 \times 10^{10}$ \\
\hline 21 & $5.143 \times 10^{7}$ & $1.803 \times 10^{8}$ & $5.916 \times 10^{8}$ & $1.832 \times 10^{9}$ & $5.383 \times 10^{9}$ & $1.51 \times 10^{10}$ & $4.059 \times 10^{10}$ & $1.05 \times 10^{11}$ \\
\hline 22 & $7.276 \times 10^{7}$ & $2.635 \times 10^{8}$ & $8.925 \times 10^{8}$ & $2.849 \times 10^{9}$ & $8.627 \times 10^{9}$ & $2.491 \times 10^{10}$ & $6.888 \times 10^{10}$ & $1.831 \times 10^{11}$ \\
\hline 23 & $1.016 \times 10^{8}$ & $3.798 \times 10^{8}$ & $1.326 \times 10^{9}$ & $4.362 \times 10^{9}$ & $1.36 \times 10^{10}$ & $4.038 \times 10^{10}$ & $1.148 \times 10^{11}$ & $3.134 \times 10^{11}$ \\
\hline 24 & $1.402 \times 10^{8}$ & $5.403 \times 10^{8}$ & $1.944 \times 10^{9}$ & $6.581 \times 10^{9}$ & $2.11 \times 10^{10}$ & $6.44 \times 10^{10}$ & $1.88 \times 10^{11}$ & $5.269 \times 10^{11}$ \\
\hline 25 & $1.913 \times 10^{8}$ & $7.595 \times 10^{8}$ & $2.813 \times 10^{9}$ & $9.794 \times 10^{9}$ & $3.227 \times 10^{10}$ & $1.012 \times 10^{11}$ & $3.031 \times 10^{11}$ & $8.715 \times 10^{11}$ \\
\hline 26 & $2.584 \times 10^{8}$ & $1.056 \times 10^{9}$ & $4.021 \times 10^{9}$ & $1.439 \times 10^{10}$ & $4.87 \times 10^{10}$ & $1.567 \times 10^{11}$ & $4.815 \times 10^{11}$ & $1.419 \times 10^{12}$ \\
\hline 27 & $3.456 \times 10^{8}$ & $1.452 \times 10^{9}$ & $5.684 \times 10^{9}$ & $2.089 \times 10^{10}$ & $7.255 \times 10^{10}$ & $2.394 \times 10^{11}$ & $7.543 \times 10^{11}$ & $2.277 \times 10^{12}$ \\
\hline 28 & $4.579 \times 10^{8}$ & $1.977 \times 10^{9}$ & $7.95 \times 10^{9}$ & $2.999 \times 10^{10}$ & $1.068 \times 10^{11}$ & $3.613 \times 10^{11}$ & $1.166 \times 10^{12}$ & $3.605 \times 10^{12}$ \\
\hline 29 & $6.017 \times 10^{8}$ & $2.668 \times 10^{9}$ & $1.101 \times 10^{10}$ & $4.259 \times 10^{10}$ & $1.555 \times 10^{11}$ & $5.389 \times 10^{11}$ & $1.781 \times 10^{12}$ & $5.634 \times 10^{12}$ \\
\hline 30 & $7.842 \times 10^{8}$ & $3.569 \times 10^{9}$ & $1.51 \times 10^{10}$ & $5.988 \times 10^{10}$ & $2.24 \times 10^{11}$ & $7.948 \times 10^{11}$ & $2.688 \times 10^{12}$ & $8.698 \times 10^{12}$ \\
\hline 31 & $1.014 \times 10^{9}$ & $4.734 \times 10^{9}$ & $2.053 \times 10^{10}$ & $8.341 \times 10^{10}$ & $3.195 \times 10^{11}$ & $1.16 \times 10^{12}$ & $4.012 \times 10^{12}$ & $1.327 \times 10^{13}$ \\
\hline 32 & $1.303 \times 10^{9}$ & $6.232 \times 10^{9}$ & $2.769 \times 10^{10}$ & $1.152 \times 10^{11}$ & $4.513 \times 10^{11}$ & $1.676 \times 10^{12}$ & $5.927 \times 10^{12}$ & $2.004 \times 10^{13}$ \\
\hline
\end{tabular}

Figure 2. Second half of the coefficients $d(n, m)$ for $m=16 \ldots 32$ of the virtual Higgs decay amplitudes $\mathcal{A}\left(h \rightarrow n \times h+m \times V_{L}\right)=n ! m ! d(n, m)(2 v)^{1-n-m}$ at the $(n+m)$-particle threshold. $\kappa=M_{W} / M_{h}=80.384 / 125.66 \simeq 0.6397$. 


\begin{tabular}{|c|c|c|c|c|c|c|c|c|c|}
\hline$a(n, m)$ & $\mathrm{m}=0$ & 2 & 4 & 6 & 8 & 10 & 12 & 14 & 16 \\
\hline $\mathrm{n}=0$ & 1 & $6.426 \times 10^{-1}$ & $6.566 \times 10^{-1}$ & $6.838 \times 10^{-1}$ & $7.196 \times 10^{-1}$ & $7.629 \times 10^{-1}$ & $8.133 \times 10^{-1}$ & $8.705 \times 10^{-1}$ & $9.352 \times 10^{-1}$ \\
\hline 1 & $7.181 \times 10^{-1}$ & 1.341 & 2.049 & 2.847 & 3.753 & 4.782 & 5.955 & 7.291 & 8.81 \\
\hline 2 & $6.783 \times 10^{-1}$ & 2.017 & 4.168 & 7.295 & $1.159 \times 10^{1}$ & $1.73 \times 10^{1}$ & $2.468 \times 10^{1}$ & $3.406 \times 10^{1}$ & $4.581 \times 10^{1}$ \\
\hline 3 & $6.521 \times 10^{-1}$ & 2.673 & 7. & $1.482 \times 10^{1}$ & $2.764 \times 10^{1}$ & $4.732 \times 10^{1}$ & $7.619 \times 10^{1}$ & $1.171 \times 10^{2}$ & $1.736 \times 10^{2}$ \\
\hline 4 & $6.327 \times 10^{-1}$ & 3.312 & $1.053 \times 10^{1}$ & $2.621 \times 10^{1}$ & $5.616 \times 10^{1}$ & $1.086 \times 10^{2}$ & $1.95 \times 10^{2}$ & $3.305 \times 10^{2}$ & $5.356 \times 10^{2}$ \\
\hline 5 & $6.175 \times 10^{-1}$ & 3.938 & $1.475 \times 10^{1}$ & $4.223 \times 10^{1}$ & $1.023 \times 10^{2}$ & $2.208 \times 10^{2}$ & $4.373 \times 10^{2}$ & $8.109 \times 10^{2}$ & $1.427 \times 10^{3}$ \\
\hline 6 & $6.05 \times 10^{-1}$ & 4.552 & $1.964 \times 10^{1}$ & $6.364 \times 10^{1}$ & $1.721 \times 10^{2}$ & $4.1 \times 10^{2}$ & $8.888 \times 10^{2}$ & $1.79 \times 10^{3}$ & $3.401 \times 10^{3}$ \\
\hline 7 & $5.944 \times 10^{-1}$ & 5.157 & $2.519 \times 10^{1}$ & $9.118 \times 10^{1}$ & $2.724 \times 10^{2}$ & $7.105 \times 10^{2}$ & $1.673 \times 10^{3}$ & $3.64 \times 10^{3}$ & $7.423 \times 10^{3}$ \\
\hline 8 & $5.853 \times 10^{-1}$ & 5.753 & $3.14 \times 10^{1}$ & $1.256 \times 10^{2}$ & $4.109 \times 10^{2}$ & $1.165 \times 10^{3}$ & $2.963 \times 10^{3}$ & $6.921 \times 10^{3}$ & $1.509 \times 10^{4}$ \\
\hline 9 & $5.773 \times 10^{-1}$ & 6.341 & $3.825 \times 10^{1}$ & $1.676 \times 10^{2}$ & $5.96 \times 10^{2}$ & $1.825 \times 10^{3}$ & $4.988 \times 10^{3}$ & $1.246 \times 10^{4}$ & $2.892 \times 10^{4}$ \\
\hline 10 & $5.702 \times 10^{-1}$ & 6.923 & $4.574 \times 10^{1}$ & $2.179 \times 10^{2}$ & $8.372 \times 10^{2}$ & $2.755 \times 10^{3}$ & $8.05 \times 10^{3}$ & $2.141 \times 10^{4}$ & $5.273 \times 10^{4}$ \\
\hline 11 & $5.637 \times 10^{-1}$ & 7.498 & $5.386 \times 10^{1}$ & $2.772 \times 10^{2}$ & $1.145 \times 10^{3}$ & $4.027 \times 10^{3}$ & $1.253 \times 10^{4}$ & $3.537 \times 10^{4}$ & $9.214 \times 10^{4}$ \\
\hline 12 & $5.579 \times 10^{-1}$ & 8.067 & $6.261 \times 10^{1}$ & $3.463 \times 10^{2}$ & $1.529 \times 10^{3}$ & $5.73 \times 10^{3}$ & $1.892 \times 10^{4}$ & $5.648 \times 10^{4}$ & $1.552 \times 10^{5}$ \\
\hline 13 & $5.526 \times 10^{-1}$ & 8.631 & $7.198 \times 10^{1}$ & $4.257 \times 10^{2}$ & $2.002 \times 10^{3}$ & $7.962 \times 10^{3}$ & $2.781 \times 10^{4}$ & $8.756 \times 10^{4}$ & $2.53 \times 10^{5}$ \\
\hline 14 & $5.477 \times 10^{-1}$ & 9.19 & $8.196 \times 10^{1}$ & $5.163 \times 10^{2}$ & $2.577 \times 10^{3}$ & $1.084 \times 10^{4}$ & $3.993 \times 10^{4}$ & $1.322 \times 10^{5}$ & $4.009 \times 10^{5}$ \\
\hline 15 & $5.431 \times 10^{-1}$ & 9.745 & $9.255 \times 10^{1}$ & $6.187 \times 10^{2}$ & $3.266 \times 10^{3}$ & $1.449 \times 10^{4}$ & $5.615 \times 10^{4}$ & $1.951 \times 10^{5}$ & $6.195 \times 10^{5}$ \\
\hline 16 & $5.389 \times 10^{-1}$ & $1.03 \times 10^{1}$ & $1.037 \times 10^{2}$ & $7.335 \times 10^{2}$ & $4.083 \times 10^{3}$ & $1.906 \times 10^{4}$ & $7.75 \times 10^{4}$ & $2.82 \times 10^{5}$ & $9.357 \times 10^{5}$ \\
\hline 17 & $5.349 \times 10^{-1}$ & $1.084 \times 10^{1}$ & $1.155 \times 10^{2}$ & $8.614 \times 10^{2}$ & $5.045 \times 10^{3}$ & $2.47 \times 10^{4}$ & $1.052 \times 10^{5}$ & $4.001 \times 10^{5}$ & $1.385 \times 10^{6}$ \\
\hline 18 & $5.312 \times 10^{-1}$ & $1.138 \times 10^{1}$ & $1.279 \times 10^{2}$ & $1.003 \times 10^{3}$ & $6.165 \times 10^{3}$ & $3.161 \times 10^{4}$ & $1.407 \times 10^{5}$ & $5.581 \times 10^{5}$ & $2.012 \times 10^{6}$ \\
\hline 19 & $5.277 \times 10^{-1}$ & $1.192 \times 10^{1}$ & $1.409 \times 10^{2}$ & $1.159 \times 10^{3}$ & $7.46 \times 10^{3}$ & $3.998 \times 10^{4}$ & $1.856 \times 10^{5}$ & $7.667 \times 10^{5}$ & $2.874 \times 10^{6}$ \\
\hline 20 & $5.244 \times 10^{-1}$ & $1.246 \times 10^{1}$ & $1.545 \times 10^{2}$ & $1.331 \times 10^{3}$ & $8.948 \times 10^{3}$ & $5.002 \times 10^{4}$ & $2.418 \times 10^{5}$ & $1.039 \times 10^{6}$ & $4.043 \times 10^{6}$ \\
\hline 21 & $5.213 \times 10^{-1}$ & $1.299 \times 10^{1}$ & $1.687 \times 10^{2}$ & $1.518 \times 10^{3}$ & $1.065 \times 10^{4}$ & $6.197 \times 10^{4}$ & $3.115 \times 10^{5}$ & $1.39 \times 10^{6}$ & $5.608 \times 10^{6}$ \\
\hline 22 & $5.183 \times 10^{-1}$ & $1.352 \times 10^{1}$ & $1.834 \times 10^{2}$ & $1.722 \times 10^{3}$ & $1.257 \times 10^{4}$ & $7.609 \times 10^{4}$ & $3.972 \times 10^{5}$ & $1.837 \times 10^{6}$ & $7.678 \times 10^{6}$ \\
\hline 23 & $5.155 \times 10^{-1}$ & $1.405 \times 10^{1}$ & $1.987 \times 10^{2}$ & $1.942 \times 10^{3}$ & $1.475 \times 10^{4}$ & $9.267 \times 10^{4}$ & $5.015 \times 10^{5}$ & $2.402 \times 10^{6}$ & $1.039 \times 10^{7}$ \\
\hline 24 & $5.128 \times 10^{-1}$ & $1.457 \times 10^{1}$ & $2.146 \times 10^{2}$ & $2.181 \times 10^{3}$ & $1.719 \times 10^{4}$ & $1.12 \times 10^{5}$ & $6.278 \times 10^{5}$ & $3.111 \times 10^{6}$ & $1.39 \times 10^{7}$ \\
\hline 25 & $5.103 \times 10^{-1}$ & $1.51 \times 10^{1}$ & $2.311 \times 10^{2}$ & $2.438 \times 10^{3}$ & $1.992 \times 10^{4}$ & $1.344 \times 10^{5}$ & $7.793 \times 10^{5}$ & $3.99 \times 10^{6}$ & $1.84 \times 10^{7}$ \\
\hline 26 & $5.078 \times 10^{-1}$ & $1.561 \times 10^{1}$ & $2.481 \times 10^{2}$ & $2.713 \times 10^{3}$ & $2.296 \times 10^{4}$ & $1.603 \times 10^{5}$ & $9.601 \times 10^{5}$ & $5.074 \times 10^{6}$ & $2.414 \times 10^{7}$ \\
\hline 27 & $5.054 \times 10^{-1}$ & $1.613 \times 10^{1}$ & $2.657 \times 10^{2}$ & $3.009 \times 10^{3}$ & $2.634 \times 10^{4}$ & $1.899 \times 10^{5}$ & $1.174 \times 10^{6}$ & $6.401 \times 10^{6}$ & $3.137 \times 10^{7}$ \\
\hline 28 & $5.032 \times 10^{-1}$ & $1.665 \times 10^{1}$ & $2.839 \times 10^{2}$ & $3.325 \times 10^{3}$ & $3.007 \times 10^{4}$ & $2.238 \times 10^{5}$ & $1.427 \times 10^{6}$ & $8.014 \times 10^{6}$ & $4.044 \times 10^{7}$ \\
\hline 29 & $5.01 \times 10^{-1}$ & $1.716 \times 10^{1}$ & $3.026 \times 10^{2}$ & $3.661 \times 10^{3}$ & $3.418 \times 10^{4}$ & $2.623 \times 10^{5}$ & $1.723 \times 10^{6}$ & $9.963 \times 10^{6}$ & $5.171 \times 10^{7}$ \\
\hline 30 & $4.989 \times 10^{-1}$ & $1.767 \times 10^{1}$ & $3.219 \times 10^{2}$ & $4.02 \times 10^{3}$ & $3.869 \times 10^{4}$ & $3.059 \times 10^{5}$ & $2.069 \times 10^{6}$ & $1.23 \times 10^{7}$ & $6.564 \times 10^{7}$ \\
\hline 31 & $4.969 \times 10^{-1}$ & $1.818 \times 10^{1}$ & $3.417 \times 10^{2}$ & $4.4 \times 10^{3}$ & $4.363 \times 10^{4}$ & $3.551 \times 10^{5}$ & $2.47 \times 10^{6}$ & $1.51 \times 10^{7}$ & $8.274 \times 10^{7}$ \\
\hline 32 & $4.949 \times 10^{-1}$ & $1.868 \times 10^{1}$ & $3.621 \times 10^{2}$ & $4.803 \times 10^{3}$ & $4.902 \times 10^{4}$ & $4.104 \times 10^{5}$ & $2.934 \times 10^{6}$ & $1.842 \times 10^{7}$ & $1.036 \times 10^{8}$ \\
\hline
\end{tabular}

Figure 3. Coefficients $a(n, m)$ for $n=0 \ldots 32$ and $m=0 \ldots 16$ of the virtual vector boson decay amplitudes $\mathcal{A}\left(V_{L} \rightarrow n \times h+(m+1) \times V_{L}\right)=n !(m+1) ! a(n, m) /(2 v)^{n+m}$ at the $(n+m+1)$-particle threshold. The value of $\kappa$ is set to the $\mathrm{SM}$ value, $\kappa=M_{W} / M_{h}=80.384 / 125.66 \simeq 0.6397$.

The scattering amplitudes on multiparticle thresholds are obtained by repeatedly differentiating the generating functions in (3.22)-(3.23) with respect to $z$ and $w^{a}$. For example, for the Higgs to $n$ Higgses and $m$ longitudinal $\mathrm{Z}$ bosons threshold amplitude we get,

$$
\mathcal{A}\left(h \rightarrow n \times h+m \times Z_{L}\right)=(2 v)^{1-n-m} n ! m ! d(n, m),
$$




\begin{tabular}{|c|c|c|c|c|c|c|c|c|}
\hline$a(n, m)$ & $\mathrm{m}=18$ & 20 & 22 & 24 & 26 & 28 & 30 & 32 \\
\hline $\mathrm{n}=0$ & $9.958 \times 10^{-1}$ & 1.066 & 1.234 & 1.042 & 2.176 & 9.866 & $-6.625 \times 10^{1}$ & $5.708 \times 10^{1}$ \\
\hline 1 & $1.054 \times 10^{1}$ & $1.248 \times 10^{1}$ & $1.466 \times 10^{1}$ & $1.663 \times 10^{1}$ & $2.117 \times 10^{1}$ & $2.508 \times 10^{1}$ & $3.169 \times 10^{1}$ & $-3.066 \times 10^{1}$ \\
\hline 2 & $6.035 \times 10^{1}$ & $7.819 \times 10^{1}$ & $9.989 \times 10^{1}$ & $1.261 \times 10^{2}$ & $1.575 \times 10^{2}$ & $1.948 \times 10^{2}$ & $2.387 \times 10^{2}$ & $2.901 \times 10^{2}$ \\
\hline 3 & $2.499 \times 10^{2}$ & $3.512 \times 10^{2}$ & $4.838 \times 10^{2}$ & $6.55 \times 10^{2}$ & $8.739 \times 10^{2}$ & $1.151 \times 10^{3}$ & $1.498 \times 10^{3}$ & $1.928 \times 10^{3}$ \\
\hline 4 & $8.369 \times 10^{2}$ & $1.269 \times 10^{3}$ & $1.875 \times 10^{3}$ & $2.711 \times 10^{3}$ & $3.847 \times 10^{3}$ & $5.371 \times 10^{3}$ & $7.388 \times 10^{3}$ & $1.003 \times 10^{4}$ \\
\hline 5 & $2.406 \times 10^{3}$ & $3.914 \times 10^{3}$ & $6.178 \times 10^{3}$ & $9.505 \times 10^{3}$ & $1.43 \times 10^{4}$ & $2.109 \times 10^{4}$ & $3.058 \times 10^{4}$ & $4.365 \times 10^{4}$ \\
\hline 6 & $6.155 \times 10^{3}$ & $1.07 \times 10^{4}$ & $1.797 \times 10^{4}$ & $2.932 \times 10^{4}$ & $4.661 \times 10^{4}$ & $7.245 \times 10^{4}$ & $1.104 \times 10^{5}$ & $1.653 \times 10^{5}$ \\
\hline 7 & $1.436 \times 10^{4}$ & $2.657 \times 10^{4}$ & $4.733 \times 10^{4}$ & $8.16 \times 10^{4}$ & $1.367 \times 10^{5}$ & $2.235 \times 10^{5}$ & $3.571 \times 10^{5}$ & $5.594 \times 10^{5}$ \\
\hline 8 & $3.108 \times 10^{4}$ & $6.099 \times 10^{4}$ & $1.149 \times 10^{5}$ & $2.088 \times 10^{5}$ & $3.678 \times 10^{5}$ & $6.303 \times 10^{5}$ & $1.054 \times 10^{6}$ & $1.725 \times 10^{6}$ \\
\hline 9 & $6.318 \times 10^{4}$ & $1.311 \times 10^{5}$ & $2.603 \times 10^{5}$ & $4.975 \times 10^{5}$ & $9.193 \times 10^{5}$ & $1.649 \times 10^{6}$ & $2.881 \times 10^{6}$ & $4.916 \times 10^{6}$ \\
\hline 10 & $1.218 \times 10^{5}$ & $2.665 \times 10^{5}$ & $5.565 \times 10^{5}$ & $1.116 \times 10^{6}$ & $2.158 \times 10^{6}$ & $4.045 \times 10^{6}$ & $7.37 \times 10^{6}$ & $1.309 \times 10^{7}$ \\
\hline 11 & $2.245 \times 10^{5}$ & $5.165 \times 10^{5}$ & $1.131 \times 10^{6}$ & $2.375 \times 10^{6}$ & $4.8 \times 10^{6}$ & $9.381 \times 10^{6}$ & $1.78 \times 10^{7}$ & $3.286 \times 10^{7}$ \\
\hline 12 & $3.976 \times 10^{5}$ & $9.599 \times 10^{5}$ & $2.202 \times 10^{6}$ & $4.828 \times 10^{6}$ & $1.018 \times 10^{7}$ & $2.071 \times 10^{7}$ & $4.085 \times 10^{7}$ & $7.83 \times 10^{7}$ \\
\hline 13 & $6.802 \times 10^{5}$ & $1.72 \times 10^{6}$ & $4.121 \times 10^{6}$ & $9.427 \times 10^{6}$ & $2.069 \times 10^{7}$ & $4.378 \times 10^{7}$ & $8.963 \times 10^{7}$ & $1.781 \times 10^{8}$ \\
\hline 14 & $1.129 \times 10^{6}$ & $2.982 \times 10^{6}$ & $7.454 \times 10^{6}$ & $1.776 \times 10^{7}$ & $4.052 \times 10^{7}$ & $8.901 \times 10^{7}$ & $1.889 \times 10^{8}$ & $3.888 \times 10^{8}$ \\
\hline 15 & $1.822 \times 10^{6}$ & $5.022 \times 10^{6}$ & $1.307 \times 10^{7}$ & $3.238 \times 10^{7}$ & $7.673 \times 10^{7}$ & $1.748 \times 10^{8}$ & $3.841 \times 10^{8}$ & $8.176 \times 10^{8}$ \\
\hline 16 & $2.872 \times 10^{6}$ & $8.241 \times 10^{6}$ & $2.231 \times 10^{7}$ & $5.736 \times 10^{7}$ & $1.409 \times 10^{8}$ & $3.324 \times 10^{8}$ & $7.558 \times 10^{8}$ & $1.662 \times 10^{9}$ \\
\hline 17 & $4.426 \times 10^{6}$ & $1.321 \times 10^{7}$ & $3.712 \times 10^{7}$ & $9.898 \times 10^{7}$ & $2.518 \times 10^{8}$ & $6.144 \times 10^{8}$ & $1.444 \times 10^{9}$ & $3.277 \times 10^{9}$ \\
\hline 18 & $6.686 \times 10^{6}$ & $2.072 \times 10^{7}$ & $6.038 \times 10^{7}$ & $1.667 \times 10^{8}$ & $4.388 \times 10^{8}$ & $1.106 \times 10^{9}$ & $2.683 \times 10^{9}$ & $6.282 \times 10^{9}$ \\
\hline 19 & $9.917 \times 10^{6}$ & $3.186 \times 10^{7}$ & $9.618 \times 10^{7}$ & $2.748 \times 10^{8}$ & $7.473 \times 10^{8}$ & $1.945 \times 10^{9}$ & $4.864 \times 10^{9}$ & $1.173 \times 10^{10}$ \\
\hline 20 & $1.447 \times 10^{7}$ & $4.814 \times 10^{7}$ & $1.503 \times 10^{8}$ & $4.437 \times 10^{8}$ & $1.246 \times 10^{9}$ & $3.344 \times 10^{9}$ & $8.617 \times 10^{9}$ & $2.14 \times 10^{10}$ \\
\hline 21 & $2.078 \times 10^{7}$ & $7.154 \times 10^{7}$ & $2.308 \times 10^{8}$ & $7.035 \times 10^{8}$ & $2.037 \times 10^{9}$ & $5.633 \times 10^{9}$ & $1.495 \times 10^{10}$ & $3.818 \times 10^{10}$ \\
\hline 22 & $2.944 \times 10^{7}$ & $1.047 \times 10^{8}$ & $3.488 \times 10^{8}$ & $1.096 \times 10^{9}$ & $3.271 \times 10^{9}$ & $9.312 \times 10^{9}$ & $2.541 \times 10^{10}$ & $6.674 \times 10^{10}$ \\
\hline 23 & $4.115 \times 10^{7}$ & $1.511 \times 10^{8}$ & $5.191 \times 10^{8}$ & $1.681 \times 10^{9}$ & $5.164 \times 10^{9}$ & $1.512 \times 10^{10}$ & $4.243 \times 10^{10}$ & $1.144 \times 10^{11}$ \\
\hline 24 & $5.684 \times 10^{7}$ & $2.152 \times 10^{8}$ & $7.619 \times 10^{8}$ & $2.54 \times 10^{9}$ & $8.027 \times 10^{9}$ & $2.417 \times 10^{10}$ & $6.964 \times 10^{10}$ & $1.928 \times 10^{11}$ \\
\hline 25 & $7.762 \times 10^{7}$ & $3.029 \times 10^{8}$ & $1.104 \times 10^{9}$ & $3.786 \times 10^{9}$ & $1.23 \times 10^{10}$ & $3.803 \times 10^{10}$ & $1.125 \times 10^{11}$ & $3.195 \times 10^{11}$ \\
\hline 26 & $1.049 \times 10^{8}$ & $4.214 \times 10^{8}$ & $1.58 \times 10^{9}$ & $5.569 \times 10^{9}$ & $1.858 \times 10^{10}$ & $5.899 \times 10^{10}$ & $1.79 \times 10^{11}$ & $5.211 \times 10^{11}$ \\
\hline 27 & $1.404 \times 10^{8}$ & $5.801 \times 10^{8}$ & $2.235 \times 10^{9}$ & $8.094 \times 10^{9}$ & $2.772 \times 10^{10}$ & $9.027 \times 10^{10}$ & $2.808 \times 10^{11}$ & $8.378 \times 10^{11}$ \\
\hline 28 & $1.861 \times 10^{8}$ & $7.905 \times 10^{8}$ & $3.129 \times 10^{9}$ & $1.163 \times 10^{10}$ & $4.086 \times 10^{10}$ & $1.364 \times 10^{11}$ & $4.348 \times 10^{11}$ & $1.328 \times 10^{12}$ \\
\hline 29 & $2.446 \times 10^{8}$ & $1.067 \times 10^{9}$ & $4.337 \times 10^{9}$ & $1.654 \times 10^{10}$ & $5.956 \times 10^{10}$ & $2.037 \times 10^{11}$ & $6.649 \times 10^{11}$ & $2.079 \times 10^{12}$ \\
\hline 30 & $3.189 \times 10^{8}$ & $1.428 \times 10^{9}$ & $5.954 \times 10^{9}$ & $2.327 \times 10^{10}$ & $8.588 \times 10^{10}$ & $3.008 \times 10^{11}$ & $1.005 \times 10^{12}$ & $3.214 \times 10^{12}$ \\
\hline 31 & $4.127 \times 10^{8}$ & $1.896 \times 10^{9}$ & $8.101 \times 10^{9}$ & $3.244 \times 10^{10}$ & $1.226 \times 10^{11}$ & $4.395 \times 10^{11}$ & $1.502 \times 10^{12}$ & $4.911 \times 10^{12}$ \\
\hline 32 & $5.301 \times 10^{8}$ & $2.497 \times 10^{9}$ & $1.093 \times 10^{10}$ & $4.482 \times 10^{10}$ & $1.733 \times 10^{11}$ & $6.357 \times 10^{11}$ & $2.221 \times 10^{12}$ & $7.422 \times 10^{12}$ \\
\hline
\end{tabular}

Figure 4. Second half of the coefficients $a(n, m)$ for $m=16 \ldots 32$ of the virtual vector boson decay amplitudes $\mathcal{A}\left(V_{L} \rightarrow n \times h+(m+1) \times V_{L}\right)$ at the $(n+m+1)$-particle threshold. As before, $\kappa=M_{W} / M_{h}=80.384 / 125.66 \simeq 0.6397$.

and for the longitudinal $\mathrm{Z}$ decaying into $n$ Higgses and $m+1$ vector bosons we have,

$$
\mathcal{A}\left(Z_{L} \rightarrow n \times h+(m+1) \times Z_{L}\right)=\frac{1}{(2 v)^{n+m}} n !(m+1) ! a(n, m) .
$$


$\mathrm{d}(\mathrm{n}, \mathrm{m})$

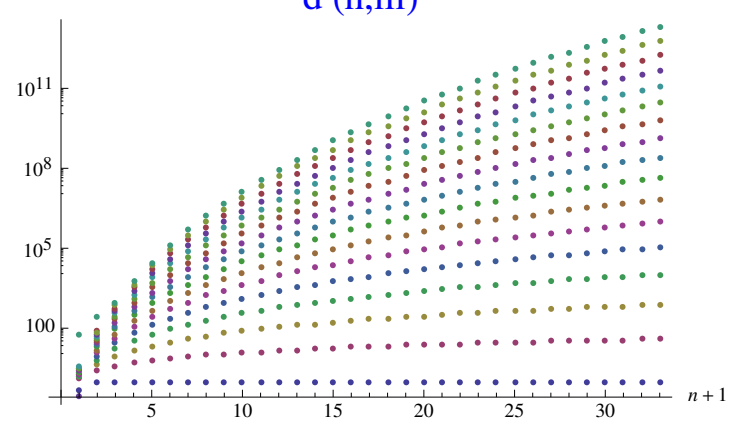

$\mathrm{a}(\mathrm{n}, \mathrm{m})$

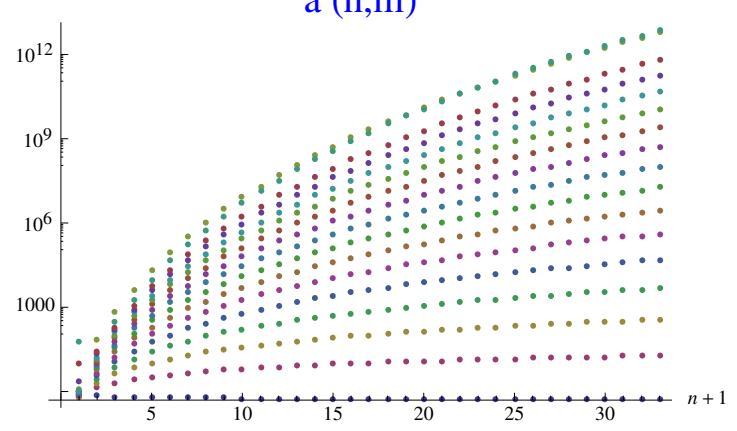

Figure 5. Coefficients $d(n, m)$ and $a(n, m)$ for generating functions of amplitudes at threshold. $\kappa=M_{W} / M_{h}=80.384 / 125.66 \simeq 0.6397$. The label $n=0 \ldots 32$ is shown along the horizontal axis and the sequences of curves correspond to $m=0,2, \ldots, 32$ from bottom to top.

The amplitudes with all varieties of $W_{L}^{ \pm}$and $Z_{L}$ in the final state, one should simply differentiate with respect to $w^{a}$ with the appropriate values of the isospin index $a=1,2,3$.

In the tables in figures $1-4$ we list the values of the coefficients $d(n, m)$ and $a(n, m)$ describing the amplitudes with up to $n=32$ Higgs bosons plus $m=32$ longitudinal vector bosons in the final state. In solving for these coefficients we have set $\kappa=M_{W} / M_{h}=$ $80.384 / 125.66 \simeq 0.6397$.

In our normalisation conventions where the generating functions and amplitudes include inverse powers of $2 v$, the coefficients of pure multi-Higgs production, $d(n, 0)$ for all $n \geq 1$, are all equal to 1 , as can be seen in the first table in figure 1 . This provides a useful reference point for the size of other generating function's coefficients. Note that by allowing the production of longitudinal vector bosons, i.e. after switching on $m>0$, the coefficients of the generating functions grow steadily with $m$, reaching $d(n, m) \sim 10^{8}$ at $m \geq 16$ and $n \geq 27$; and $d(n, m) \sim 10^{13}$ at $m=32$ and $n=31$, cf. the table in figure 1 . Similar growth with $m$ occurs for the $a(n, m)$ coefficients of the gauge field generating function, see tables in figures 3-4. This numerical growth of the coefficients is in addition to the multiplicative $n$ ! and $m$ ! factors in the amplitudes in (3.24)-(3.25).

In figure 5 we show the logarithmic plots of all $d(n, m)$ and $a(n, m)$ for $n=0 \ldots 32$ and $m=0,2, \ldots, 32$. These plots can be interpreted as sequences of curves, each curve representing $d(n, m)$ and $a(n, m)$ as functions of $n$ for a fixed value of $m$. Increasing values of $m=0,2, \ldots, 32$ corresponds to moving upwards from lower to higher curves.

For the relative comparison between the coefficients it might be more appropriate to normalise vector boson and Higgs legs by their respective masses rather than a universal factor of $2 v$. This would be characterised by rescaling the coefficients by $\kappa^{m}$. These are shown in figure 6 , from which we see that there is still more than six orders of magnitude growth in coefficients as one increases $m$ to $\sim 30$.

The importance of multiple vector boson emissions relative to the multi-Higgs production should decrease as one decreases the vector boson mass relative to the Higgs mass, so that in the asymptotic case of $M_{V}=0$ we are left only with $d(n, 0)$, as the classical equations would dictate. This is indeed the case as can be seen from figure 7 which shows 

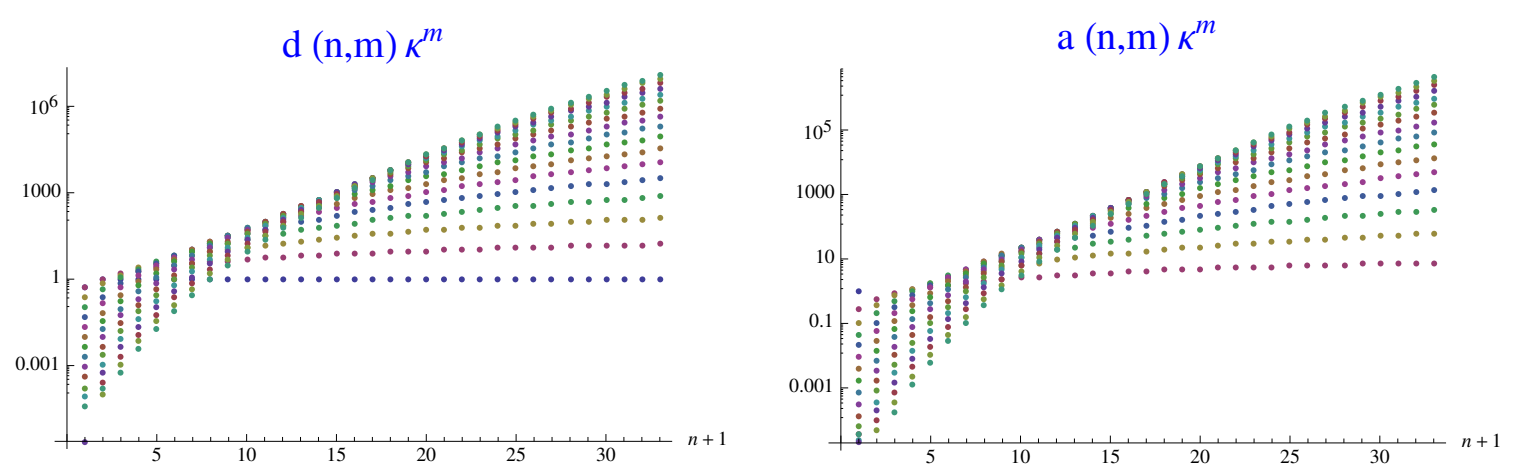

Figure 6. Amplitude coefficients of figure 5 rescaled by $\kappa^{m}$ (where $\kappa \simeq 0.6397$ ).
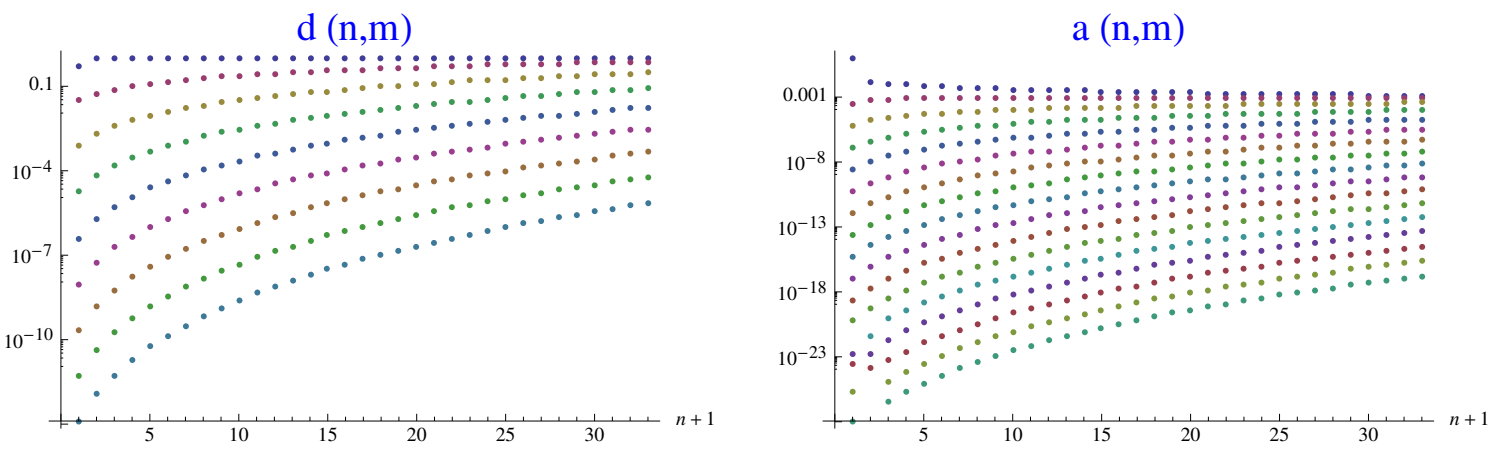

Figure 7. The light vector boson case, $M_{V} \ll M_{h}$, with the non-SM choice $\kappa=M_{V} / M_{h}=0.1$. The amplitude coefficients $d(n, m)$ and $a(n, m)$ are shown as functions of $n=0 \ldots 32$. The sequences of curves correspond to $m=0,2, \ldots, 32$ from top to bottom.

the (unrescaled) coefficients $d(n, m)$ and $a(n, m)$ for the case of the vector boson taken to be 10 times lighter than the Higgs. In this case, the coefficients actually steadily decrease when one increases $m$.

\section{Conclusions}

In the spontaneously broken gauge theory we have computed tree-level amplitudes for production of high multiplicities of longitudinal vector bosons and Higgs bosons at threshold. We found that these amplitudes grow factorially $\sim m ! n$ ! with the numbers of vector and Higgs bosons in the final state, thus extending the known factorial growth in scalar QFT to the massive Gauge-Higgs theory. We have also shown that in addition to the $m ! n$ ! factorial growth, the amplitudes involving high multiplicities of longitudinal vector bosons grow faster than the amplitudes with only the multiple Higgs production.

These findings imply that broken gauge theory shows a very different perturbative behaviour at high multiplicities from massless gauge theories, such as QCD. In the latter case the factorial growth of the number of Feynman diagrams is not inherited by multigluon amplitudes, as can be seen from e.g. the applications of MHV rules [26] or the BCFW recursion relations [27]. 
Before we conclude we would like to comment on the limitations, extensions and implications of these results.

1. In this paper we have not considered the production of transversely polarised massive vector bosons. This task can be approached by solving iteratively the full eq. (3.7) including the commutator term. It would be interesting to find out whether or not the production of $m_{T}$ transverse vector bosons shows any signs of growth with respect to $m_{T}$ and to compare this behaviour with QCD. In either case, at tree-level this would not affect the factorial growth of longitudinal vector and Higgs production found here.

2. What about higher loop corrections to our tree-level amplitudes at threshold? A simple scaling estimate of of the size of one-loop correction to an $n$-point amplitude in a generic theory is $\mathcal{A}_{n}^{1-\text { loop }} \sim \alpha^{n} n^{2} \mathcal{A}_{n}^{\text {tree }}$, where the factor of $n^{2}$ comes from the number of ways one can attach an internal propagator between $n$ external legs, and $\alpha$ represents the generic coupling constant $\left(\alpha_{W}\right.$ or $\left.\lambda\right)$. In scalar field theory this scaling argument was confirmed in $[8,9]$ by computing the 1-loop tadpole graph in the background of the classical generating function (2.10) or (2.14). For example, the 1-loop corrected threshold amplitude in the real scalar QFT (2.12) is given by [9]

$$
\phi^{4} \text { theory eq. (2.12): } \quad \mathcal{A}_{1 \rightarrow n}^{\text {tree+1loop }}=n !(2 v)^{1-n}\left(1+n(n-1) \frac{\sqrt{3} \lambda}{8 \pi}\right) .
$$

The $\left(n^{2} \times\right.$ coupling constant) behaviour of the loop corrections does not eliminate the factorial growth found at tree-level, in fact at the interesting for us multiplicities, $n \sim 1 / \lambda \sim$ $1 / \alpha_{W}$, it indicates a complete breakdown of the fixed order perturbation theory for high multiplicity amplitudes at threshold. Based on the rather general nature of the scaling argument, we expect that this conclusion also applies to the massive Gauge-Higgs theory.

There are strong indications, based on the analysis of leading singularities of the multiloop expansion around singular generating functions in scalar field theory, that the 1-loop correction exponentiates [10],

$$
\mathcal{A}_{1 \rightarrow n}=\mathcal{A}_{1 \rightarrow n}^{\text {tree }} \times \exp \left[B \lambda n^{2}+\mathcal{O}(\lambda n)\right]
$$

in the limit $\lambda \rightarrow 0, n \rightarrow \infty$ with $\lambda n^{2}$ fixed. Here $B$ is the constant factor determined from the 1-loop calculation,

$$
\begin{aligned}
\phi^{4} \text { theory eq. (2.1): } & B=-\frac{1}{64 \pi^{2}}(\log (7+4 \sqrt{3})-i \pi), \\
\phi^{4} \text { theory eq. (2.12) }: & B=+\frac{\sqrt{3}}{8 \pi},
\end{aligned}
$$

where the last equation is in agreement with (4.1) and leads to the exponential enhancement of the tree-level threshold amplitude at least in the leading order in $n^{2} \lambda$. It would be interesting to investigate how the vector boson emission and loops would affect this result and if the overall sign of $\operatorname{Re}(B)$ in the Gauge-Higgs theory will remain positive. Of course, the higher-order corrections $\sim n \lambda$ are also important, as we are interested in multiplicities $n \sim 1 / \lambda$. 
3. Going near or off the threshold. Remarkable progress has been made in the mid 90's in understanding the scalar QFT case, see [14] for a review of these developments. To characterise the behaviour of the amplitude off the threshold it is convenient to define the average kinetic energy per particle (per mass) in the final state [10], which in the non-relativistic limit near the threshold becomes,

$$
\varepsilon:=\frac{E-n M}{n M} \rightarrow \frac{1}{2 n M^{2}} \sum_{j=1}^{n} \vec{p}_{j}^{2} .
$$

In the non-relativistic multi-particle limit $n \rightarrow \infty, \varepsilon \rightarrow 0$ with $\varepsilon n$ fixed, one can solve the recursion relations for the scattering amplitudes, but now incorporating the dependence on $\varepsilon$. For the simple $\phi^{4}$ theory (2.1), the result is [10] (cf. (2.11)),

$$
\mathcal{A}_{1 \rightarrow n}=n !\left(\frac{\lambda}{8 M^{2}}\right)^{\frac{n-1}{2}} \times \exp \left[-\frac{5}{6} n \varepsilon\right],
$$

which solves the recursion relations to order $\varepsilon$.

Combining these results for the off-threshold non-relativistic amplitudes (4.6) and the exponentiation of the 1-loop corrections (4.2), the authors of [14] have argued that the multi-particle cross-sections in the case of scalar field theory take the form,

$$
\sigma_{n} \sim \exp \left[\lambda^{-1} F(\lambda n, \epsilon)\right] .
$$

At tree-level the 'holy-grail' function $F$ in the model (2.1) takes the form [10, 14],

$$
\lambda^{-1} F^{\text {tree }}=n\left[\log \left(\frac{\lambda n}{16}\right)-1\right]+n \frac{3}{2}\left[\log \left(\frac{\varepsilon}{3 \pi}\right)+1\right]-n \varepsilon \frac{17}{12} .
$$

What is particularly important for our purposes of understanding the role played by the amplitudes on threshold in the more general theory, such as the Gauge-Higgs theory considered in this paper, is that all terms on the r.h.s. of (4.8) have a clear physical interpretation in terms of the original tree-level threshold amplitude. The first term in square brackets is the logarithm of the tree-level squared amplitude at threshold, $\left|\mathcal{A}_{1 \rightarrow n}^{\text {tree }}\right|^{2}$. The second term is the result of integrating this constant amplitude over the non-relativistic $n$-particle phase space. Finally, the third term on the r.h.s. of (4.8) is the correction coming from integrating the $\varepsilon$-dependent non-relativistic expression (4.6).

It is then natural to conjecture that the in our Gauge-Higgs theory the high-multiplicity tree-level cross-section (e.g. with the virtual Higgs in the intermediate state) take the form,

$$
\begin{aligned}
\log \sigma_{n+m}^{\text {tree }} \sim & 2 \log (d(n, m))+n\left[\log \left(\frac{\lambda n}{4}\right)-1\right]+m\left[\log \left(4 g^{2} n\right)-1\right] \\
& +n \frac{3}{2}\left[\log \left(\frac{\varepsilon_{h}}{3 \pi}\right)+1\right]+m \frac{3}{2}\left[\log \left(\frac{\varepsilon_{V}}{\pi}\right)+1\right]+\mathcal{O}\left(n \varepsilon_{h}+m \varepsilon_{V}\right),
\end{aligned}
$$

where $\varepsilon_{h}$ and $\varepsilon_{V}$ are the non-relativistic kinetic energies of the $n$ Higgs bosons and $m$ longitudinal vector bosons in the final state,

$$
\varepsilon_{h}=\frac{1}{2 n M_{h}^{2}} \sum_{j=1}^{n} \vec{p}_{j}^{2}, \quad \varepsilon_{V}=\frac{1}{2 n M_{V}^{2}} \sum_{k=1}^{m} \vec{p}_{k}^{2} .
$$


One can also consider computing the off-shell Gauge-Higgs amplitudes directly. The recent progress in scattering amplitudes calculations in gauge theory based on on-shell methods is largely reliant on massless states. These methods were extended to incorporate one or few massive stats: the Higgs boson in [28], the massive vector boson currents in [29] and few massive particles in the BCFW rules in [30,31]. Nevertheless, these results do not capture directly the high-multiplicity production of massive states we are interested in in the broken gauge theory.

One promising way forward would be to use the approach developed in [32] for computing colour-ordered amplitudes in the broken gauge theory. Combining colour-ordering with the Berends-Giele-type recursion relations, the authors of [32] have computed numerically tree-level scattering amplitudes of up to 9 massive vector bosons (with generic polarisations, in a generic kinematics, and no Higgses). It should be possible to extend these results to $\sim 20-30$ gauge and Higgs bosons with external momenta near (and also away from) the threshold.

For the case of the scalar theory with SSB (2.14) it was advocated in [11] that the $1 \rightarrow n$ process should proceed via producing a non-perturbative bubble in the intermediate state, $1 \rightarrow n=1 \rightarrow B \rightarrow n$, with the bubble $B$ developing an exponentially damping formfactor as soon as the 3-momenta of external states exceed the inverse radius of the bubble. The bubble interpretation was based on the fact that in Euclidean time the generating function (2.14) is the kink solution of the model. The conclusion of [11] was that the bubble form-factor suppression would lead to a non-perturbative exponential suppression of the $1 \rightarrow n$ processes in this theory. In the Gauge-Higgs theory the generating function does not have a kink form as soon as the vector boson fields are taken into account. The imaginary-time solution to (3.9) is more of the free-fall type - not unlike the solution (2.10) which reaches infinite field values in finite time. This then also affects the Higgs field in (3.8). We do not expect that in the Gauge-Higgs theory the high-multiplicity process would proceed though a non-singlular and relatively long-lived semi-classical state (which the bubble was in the scalar SSB case) to provide for a sharp semi-classical form-factor which would suppress processes of the threshold.

4. The main conclusion we want to draw from the computations presented in this paper is that the energies which should be available at the next generation of $p p$ colliders, will kinematically allow for sufficiently high multiplicities of Higgses and longitudinal $W$ 's and $Z$ 's production where the electroweak sector of the Standard Model becomes strong and the perturbation theory breaks down. To answer the question whether these very high multiplicity processes become observable and even unsuppressed requires physics beyond perturbation theory in the weak sector. This is an interesting point on its own right, as it is often generally assumed that collider phenomenology of the electroweak SM sector is always perturbative.

5. Finally, as already mentioned, this 'perturbative' high-multiplicity $n \sim 1 / \alpha_{W}$ production in the topologically trivial sector is a logical counterpart of the more complicated instanton-induced non-perturbative $B-L$ processes, which also require $n \sim 1 / \alpha_{W}$ electroweak quanta in final states. There is a degree of complementarity between these two processes, as one would expect that if the non-perturbative $B-L$ processes passing over 
the sphaleron barrier become observable, the complementary perturbative processes at same energies and multiplicities should become strong (or non-perturbative). Also it is worthwhile pointing out that the phenomenological collider signatures of the final state for these two types of processes would be largely indistinguishable unless one measures the net charge of electrically charged leptons in the final state. If it will turn out that the very high multiplicity processes in the topologically trivial sector become observable, there will be fewer theoretical obstacles for the B-L processes overcoming their exponential suppression in high energy collisions.

\section{Acknowledgments}

I am grateful to Jeppe Andersen, Nima Arkani-Hamed, Tao Han, Michelangelo Mangano and Andreas Ringwald for useful discussions of perturbative and non-perturbative aspects of electroweak dynamics at $\sim 100 \mathrm{TeV}$. This work started at the 'BSM prospects for Future Circular Colliders' meeting at CERN and the CFHEP Symposium in Beijing. The research is supported by STFC, the Wolfson Foundation and Royal Society.

Open Access. This article is distributed under the terms of the Creative Commons Attribution License (CC-BY 4.0), which permits any use, distribution and reproduction in any medium, provided the original author(s) and source are credited.

\section{References}

[1] F.J. Dyson, Divergence of perturbation theory in quantum electrodynamics, Phys. Rev. $\mathbf{8 5}$ (1952) 631 [INSPIRE].

[2] J.M. Cornwall, On the High-energy Behavior of Weakly Coupled Gauge Theories, Phys. Lett. B 243 (1990) 271 [INSPIRE].

[3] H. Goldberg, Breakdown of perturbation theory at tree level in theories with scalars, Phys. Lett. B 246 (1990) 445 [INSPIRE].

[4] M.B. Voloshin, Multiparticle amplitudes at zero energy and momentum in scalar theory, Nucl. Phys. B 383 (1992) 233 [INSPIRE].

[5] E.N. Argyres, R.H.P. Kleiss and C.G. Papadopoulos, Amplitude estimates for multi - Higgs production at high-energies, Nucl. Phys. B 391 (1993) 42 [INSPIRE].

[6] L.S. Brown, Summing tree graphs at threshold, Phys. Rev. D 46 (1992) 4125 [hep-ph/9209203] [INSPIRE].

[7] M.B. Voloshin, Estimate of the onset of nonperturbative particle production at high-energy in a scalar theory, Phys. Lett. B 293 (1992) 389 [INSPIRE].

[8] M.B. Voloshin, Summing one loop graphs at multiparticle threshold, Phys. Rev. D 47 (1993) 357 [hep-ph/9209240] [INSPIRE].

[9] B.H. Smith, Summing one loop graphs in a theory with broken symmetry, Phys. Rev. D 47 (1993) 3518 [hep-ph/9209287] [INSPIRE].

[10] M.V. Libanov, V.A. Rubakov, D.T. Son and S.V. Troitsky, Exponentiation of multiparticle amplitudes in scalar theories, Phys. Rev. D 50 (1994) 7553 [hep-ph/9407381] [InSPIRE]. 
[11] A.S. Gorsky and M.B. Voloshin, Nonperturbative production of multiboson states and quantum bubbles, Phys. Rev. D 48 (1993) 3843 [hep-ph/9305219] [INSPIRE].

[12] D.T. Son, Semiclassical approach for multiparticle production in scalar theories, Nucl. Phys. B 477 (1996) 378 [hep-ph/9505338] [INSPIRE].

[13] F.L. Bezrukov, M.V. Libanov and S.V. Troitsky, O(4) symmetric singular solutions and multiparticle cross-sections in $\phi^{4}$ theory at tree level, Mod. Phys. Lett. A 10 (1995) 2135 [hep-ph/9508220] [INSPIRE].

[14] M.V. Libanov, V.A. Rubakov and S.V. Troitsky, Multiparticle processes and semiclassical analysis in bosonic field theories, Phys. Part. Nucl. 28 (1997) 217 [INSPIRE].

[15] S.J. Parke and T.R. Taylor, An Amplitude for $n$ Gluon Scattering, Phys. Rev. Lett. 56 (1986) 2459 [INSPIRE].

[16] M.L. Mangano and S.J. Parke, Multiparton amplitudes in gauge theories, Phys. Rept. 200 (1991) 301 [hep-th/0509223] [INSPIRE].

[17] L.J. Dixon, Calculating scattering amplitudes efficiently, hep-ph/9601359 [INSPIRE].

[18] A. Ringwald, High-Energy Breakdown of Perturbation Theory in the Electroweak Instanton Sector, Nucl. Phys. B 330 (1990) 1 [InSPIRE].

[19] L.D. McLerran, A.I. Vainshtein and M.B. Voloshin, Electroweak Interactions Become Strong at Energy Above Approximately 10-TeV, Phys. Rev. D 42 (1990) 171 [inSPIRE].

[20] V.V. Khoze and A. Ringwald, Total cross-section for anomalous fermion number violation via dispersion relation, Nucl. Phys. B 355 (1991) 351 [INSPIRE].

[21] V.V. Khoze and A. Ringwald, Nonperturbative contribution to total cross-sections in nonAbelian gauge theories, Phys. Lett. B 259 (1991) 106 [INSPIRE].

[22] S.Y. Khlebnikov, V.A. Rubakov and P.G. Tinyakov, Instanton induced cross-sections below the sphaleron, Nucl. Phys. B 350 (1991) 441 [INSPIRE].

[23] M.P. Mattis, The Riddle of high-energy baryon number violation, Phys. Rept. 214 (1992) 159 [INSPIRE].

[24] F.L. Bezrukov, D. Levkov, C. Rebbi, V.A. Rubakov and P. Tinyakov, Semiclassical study of baryon and lepton number violation in high-energy electroweak collisions, Phys. Rev. D 68 (2003) 036005 [hep-ph/0304180] [INSPIRE].

[25] F.L. Bezrukov, D. Levkov, C. Rebbi, V.A. Rubakov and P. Tinyakov, Suppression of baryon number violation in electroweak collisions: Numerical results, Phys. Lett. B 574 (2003) 75 [hep-ph/0305300] [INSPIRE].

[26] F. Cachazo, P. Svrček and E. Witten, MHV vertices and tree amplitudes in gauge theory, JHEP 09 (2004) 006 [hep-th/0403047] [INSPIRE].

[27] R. Britto, F. Cachazo, B. Feng and E. Witten, Direct proof of tree-level recursion relation in Yang-Mills theory, Phys. Rev. Lett. 94 (2005) 181602 [hep-th/0501052] [INSPIRE].

[28] L.J. Dixon, E.W.N. Glover and V.V. Khoze, MHV rules for Higgs plus multi-gluon amplitudes, JHEP 12 (2004) 015 [hep-th/0411092] [INSPIRE].

[29] Z. Bern, D. Forde, D.A. Kosower and P. Mastrolia, Twistor-inspired construction of electroweak vector boson currents, Phys. Rev. D 72 (2005) 025006 [hep-ph/0412167] [INSPIRE]. 
[30] S.D. Badger, E.W.N. Glover, V.V. Khoze and P. Svrček, Recursion relations for gauge theory amplitudes with massive particles, JHEP 07 (2005) 025 [hep-th/0504159] [INSPIRE].

[31] S.D. Badger, E.W.N. Glover and V.V. Khoze, Recursion relations for gauge theory amplitudes with massive vector bosons and fermions, JHEP 01 (2006) 066 [hep-th/0507161] [INSPIRE].

[32] L. Dai, K. Melnikov and F. Caola, Tree amplitudes and color decomposition in broken SU(2), JHEP 04 (2012) 095 [arXiv: 1201.1523] [INSPIRE]. 